\title{
Facial Nerve Innervating Pinnae Muscles of the Gerbil: Three-Dimensional Construction with Respect to Neighboring Structures
}

\author{
Akiko Seto-Ohshima ${ }^{1}$, Yoshiya Murashima ${ }^{2}$, Noriko Kawamura ${ }^{1}$, Takayuki Aoi ${ }^{1}$ \\ and Muneyuki Ito ${ }^{1}$
}

\begin{abstract}
'Institute for Developmental Research, Aichi Human Service Center, Kasugai 480-0392 and 2Department of Neurophysiology, Tokyo Institute of Psychiatry, Tokyo 156-0057
\end{abstract}

Received for publication December 2, 1997 and in revised form January 20, 1998

\begin{abstract}
The Mongolian gerbil Meriones unguiculatus) is an animal model of epilepsy in which the epileptic behaviors develop along with chronological development. In this study, to clarify the mechanism of epileptogenesis in the gerbil, the developmental change of the behaviors elicited by posture change was followed in the previously established animals of a seizure-sensitive strain and a -resistant one. Posture change is a strong inducer of seizure in the adult animals of the sensitive strain. The results show that a bilateral and synchronized rhythmical movement of the pinnae was induced in the young animals of the sensitive strain, which seemed to correspond to an earlier stage, if not the first, in the establishment of epileptogenesis. In contrast, posture change did not induce either the adult-type seizure or the characteristic move-

ment of the pinnae in the animals of the resistant strain. As the first step in clarifying the reason for this difference between these two strains, we studied the structure of the facial nerve which innervates the muscle of the pinnae, using consecutive paraffin sections of decalcified heads of both strains.

In both strains, the facial nerve left the brainstem, ran laterally along the vestibular nerve bundle and at the geniculate ganglion, it changed its direction posteriorly and laterally. When it left the skull, it changed its direction again and descended ventrally, extending a branch to the ear on its way. In the seizure-resistant gerbils, this branch entered the muscle in a similar way to the seizure-sensitive animals. Therefore, these two strains probably have difference(s) in other components although the possibility of a functional difference in the facial nerve remains.
\end{abstract}

Key words: Gerbil, Facial nerve, Seizure

\section{Introduction}

During chronological development, Mongolian gerbil (Meriones unguiculatus) shows a stimulus-induced epileptic motor manifestation that develops around 2 months of age as myoclonus, progressing to a generalized tonicclonic convulsion. This epileptic disorder is considered to correspond to the idiopathic generalized epilepsies of humans [7]. The threshold for seizures varies with individuals and the participation of genetic factors for determination of seizure susceptibility has been considered. To date, some abnormalities in the nervous system of the seizure-sensitive gerbils have been reported [see, 7]. However, one of them was denied recently [3] and the

Correspondence to: Dr. Akiko Seto-Ohshima, Institute for Developmental Research, Aichi Human Service Center, 713-8 Kamiyacho, Kasugai, Aichi 480-0392, Japan. exact mechanism of epileptogenesis under genetic control is still unknown.

Previously, at the Institute for Developmental Research, we established a seizure-sensitive strain (MGS/Idr) by selectively inbreeding gerbils for spontaneous motor seizures elicited by posture change [14]. In this strain, all animals exhibit seizures. Developmental change of the epileptic behaviors was followed in this strain and it was found that the seizure developed from Stages 1 to 3 which we denoted at the stages before full seizure appeared [14]. This progressive nature of epileptic behaviors led us to the idea that the development of the seizure in the gerbil may have some resemblance to the "kindling model" in which a brief stimulation makes the brain progressively epileptic when stimulation is repeatedly given [4]. Taking this into consideration, some steps might be present before Stage 1, since in this stage where the animals stopped at one place with flattened ears, twitch- 
ed the vibrissae and closed the eyes, several parts of the body are already included in the epileptic motor manifestation. Careful observation of the behaviors elicited in young sensitive animals was expected to give some information on the formation and development of the epileptic motor manifestations.

In this study, we followed the early behaviors elicited by posture change in the young animals of the sensitive strain, compared it with those of a seizure-resistant strain established at Tokyo Institute of Psychiatry by the selective inbreeding for resistance to a different kind of stimulation [17] and as the first step in obtaining some information on the different behaviors observed, that is, the absence of the movement of the pinnae of the seizureresistant animals, the macroscopical structure of the facial nerve of these two strains was studied.

\section{Materials and Methods}

\section{Experimental animals and behavioral study}

For this study, the animals of the seizure-sensitive strain, MGS/Idr and those of the seizure-resistant strain were maintained at the Institute for Developmental Research. They were bred with free access to pelleted food and water, with constant temperature $\left(24^{\circ} \mathrm{C}\right)$ and humidity (50\%). Light was provided between $06: 00$ and $18: 30 \mathrm{~h}$.

The selection method for the seizure-sensitive strain (ss) was as described previously [13], that is, to suspend the animal by its tail and press its back for $5 \mathrm{sec}$ (S-method). This posture change was found to be a potent inducer of epileptic seizures in this strain. The seizure-resistant strain (sr) was established at the Tokyo Institute of Psychiatry by the selective inbreeding for resistance to the stimulation given by the following procedure (R-method); (1) Place the animals on a wire net $(50 \times 50 \mathrm{~cm})$ and observe for $3 \mathrm{~min}$. (2) If the seizure is not induced, place the animals in a box $(21 \mathrm{~cm} \times 21 \mathrm{~cm} \times 21 \mathrm{~cm})$ and observe for $1 \mathrm{~min}$. (3) If the seizure is not induced, then toss the animals 20 times in the box and observe the behavior. This method had been used for usual characterization of this strain to ascertain this phenotype.

For this study, the individual animals were subjected to the S-method once a week when their cages were changed and the resultant behaviors were recorded. The developmental change of behaviors induced by this method was followed using 135 ss animals ( 76 female and 59 male) and 26 sr animals (17 female and 9 male).

In some experiments, the R-method in which the size of the wire net used was slightly different from the original one $(40 \times 60 \mathrm{~cm})$ was also applied as described in "Results".

\section{Morphological study}

For the macroscopical study of the facial nerve which innervates the ear muscle, 3 animals of both strains at 5560 days of age were used. The ss animals used had shown Stage 1 behavior [14] after being subjected to the
S-method. They were anesthetized using sodium pentobarbital $(75 \mathrm{mg} / \mathrm{g}$ body weight), then perfused transcardially with physiological saline, followed by $4 \%$ paraformaldehyde in $0.1 \mathrm{M}$ phosphate buffer ( $\mathrm{pH} 7.4)$. The skin of the head was mostly peeled away except for the area around the ear. The mandibula with surrounding muscle including the tongue were removed. The treated heads were fixed for 2 more days in an identical fixative at $4^{\circ} \mathrm{C}$, washed with running water, treated for $1 \mathrm{hr}$ with $70 \%$ alcohol and then immersed in $10 \%$ formic acid at $37^{\circ} \mathrm{C}$ as described previously $[8,9]$. When the bones were soft enough to be cut easily with a razor, a frontal cut at the level of the superior colliculus and a sagittal cut at the midline were made on each specimen. These blocks were processed for paraffin embedding. The left brain was used for horizontal sections. The right brain was used for frontal sections ( 2 brains) or sagittal sections (1 brain). The consecutive sections ( $7 \mu \mathrm{m}$ thick) from each specimen were mounted on glass slides and stained using mainly the Klüver-Barrera (KB) method which stains the myeline sheath blue and the nucleus and the Nissle substance of the neurons violet [12]. The cartilage was also stained a clear rose-color using this method. Some remaining sections were stained with hematoxylin and eosin (HE).

A three-dimensional profile of the facial nerve near the posterior auricle muscle was reconstructed using 25 and 32 horizontal sections, having an interval of $35 \mu \mathrm{m}$, for the ss and sr animals, respectively. They were photographed and the outline of the facial nerve, the posterior auricle muscle and the cartilage of the ear was traced on OHP paper. These papers were stacked in order, maintaining the $\mathrm{z}$-axis and then were moved along the anterior-posterior axis of the figure, by a constant length, one by one. Connecting of the shifted outline produced a three-dimensional image.

To produce a computer-assisted three-dimensional reconstruction showing the spacial relationship between the vestibular nerve and the facial nerve, the figures of 18 consecutive horizontal sections of an ss animal were photographed and the outline of the vestibular nerve, the macula of utricle, the ampullary crista of the lateral semicircular canal and the facial nerve in each figure were traced on OHP paper. These papers were stacked in order, maintaining the z-axis. On each paper, 4 reference points were plotted through the series. Then, these figures were digitized using a scanner (ScanJet 4c/T, HewlettPackard Company, CO, U.S.A.) and processed using the programs, "Photoshop" (Adobe System Inc., CA, U.S.A.) and "IPLab Spectrum" (Scanalytics, Inc., U.S.A.). The three-dimensional structure was reconstructed with the program, "VoxBlast" (Vaytek Inc., U.S.A.). The computers used were Power Macintosh 6100/66 and Power Macintosh 8500/132 (Apple Computer, Inc., CA, U.S.A.).

Some photographs were digitized with a film scanner (QuickScan 35, Minolta Co., Tokyo, Japan) and processed using the program "Photoshop". The figure was 
printed out on OHP paper with a color printer (PM-700C, Epson, Tokyo, Japan) and used to obtain an image of the spatial relationship between the facial nerve bundle and the vestibular nerve bundle and the vestibular ganglion.

For a retrograde tracing experiment with horseradish peroxidase (HRP), the ss animals were anesthetized using sodium pentobarbital $(75 \mathrm{mg} / \mathrm{g}$ body weight) and a cut was made in the skin of the posterior half area around a pinnae. The muscle was carefully removed and at the location where a round lymph node was found, an aliquot of horseradish peroxidase solution $(2.4 \mathrm{mg}$ peroxidase $/ 10 \mu \mathrm{l}$ of sterilized $0.9 \% \mathrm{NaCl}, 4-6 \mu \mathrm{l} / \mathrm{animal}$ ) was slowly injected into the area where the posterior auricle branch of the facial nerve was expected to turn into the ear muscle. After injection, a small amount of Vaseline (white) (Tokai Seiyaku, Nagoya, Japan) was applied to prevent the spread of HRP into surrounding areas as in [14] and the cut in the skin was sutured together. At 4 or $24 \mathrm{hr}$ after operation, the animals were anesthetized again and perfused sequentially with saline, $80 \mathrm{ml}$ of fixative containing $1 \%$ paraformaldehyde and $1.25 \%$ glutaraldehyde in $0.1 \mathrm{M}$ phosphate buffer ( $\mathrm{pH} \mathrm{7.4)}$ and $30 \mathrm{ml}$ of cold $4 \%$ paraformaldehyde in the aforementioned buffer. The brains were removed, further fixed for $2 \mathrm{hr}$ and then transferred into $10 \%$ sucrose in the above buffer followed by $20 \%$ sucrose in the same buffer. Consecutive sagittal sections $(90 \mu \mathrm{m}$ thick) were made on a freezing-microtome and stained for peroxidase activity by essentially the same method as in [1]. The stained sections were placed on gelatin-coated slides, cleared with xylene and coverslipped with an embedding medium.

\section{Electrical stimulation of the muscle}

To investigate which muscle responds to the backward movement of the pinnae, the 4 ss animals in which the rhythmical ear movement had been induced were anesthetized using sodium pentobarbital $(75 \mathrm{mg} / \mathrm{g}$ body weight) and the muscles around the ear hole were stimulated electrically with 30 rectangular pulses of which duration time and the interval were $500 \mu \mathrm{sec}$ and $27 \mathrm{msec}$, respectively. For stimulation, two needles $(26 \mathrm{G})$ connected to coated stainless wires and a stimulator (SEN-7203, Nihon Koden, Tokyo, Japan) connected to an isolator (SS-202J, Nihon Koden, Tokyo, Japan) were used. The voltage was increased until movement of the ear was elicited. Ordinarily, it ranged $0.6-3 \mathrm{~V}$.

\section{Results}

Developmental analysis of the behavior elicited in the ss strain by stimulation using the S-method revealed that bilateral and synchronized rhythmical backwards movement of the pinnae was the first detectable behavior (Step 1) as shown in Fig. 1. It usually started just after the animals were replaced in the cage, although it was occasionally noticed that the movement started while the animals were suspended in their tails and when the hanging time was extended. The average frequency of the rhythm was $1.46 / \mathrm{sec}(\mathrm{n}=9 ; 1.29-1.64 / \mathrm{sec})$ and the movement continued for from several to ten or more seconds. While exhibiting the rhythmical ear movement, the animals appeared to walk normally. This movement was detected in $84 \%$ female and $78 \%$ male animals in this series.

The behaviors elicited by the S-method applied once a week developed one by one as follows. Step 2: The animals walked apparently normally but the head and the neck moved rhythmically at a frequency similar to that of the ear movement in Step 1. The ears also moved. Step 3: The animals stopped and exhibited movement of the head and neck at a frequency still similar to that in Steps 1 and 2. Step 4: The rhythmical movement increased in speed and the ears were continuously flattened backwards. The eyes closed and the vibrissae vibrated very rapidly. It is a shorter version of Stage I. Step 5:

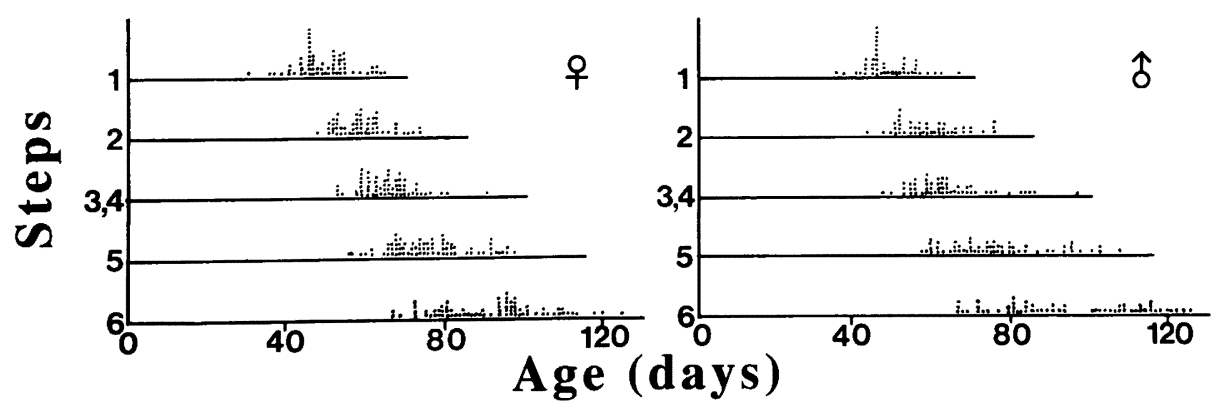

Fig. 1. Developmental profile of the behavior elicited by posture change. The animals were subjected to the $\mathrm{S}$ method as described in "Materials and Methods". Based on the developmental records of the resultant behaviors of individuals, the animals are plotted against the date at which they first showed the behavior of each step (see "Results"). One dot indicates one animal. Step 1: Bilateral and synchronized rhythmical backwards movement of the pinnae was observed. Step 2: The animals walked apparently normally but the ears, head and neck moved rhythmically at a frequency similar to that of the ear movement in Step 1. Step 3: The animals stopped and exhibited a localized movement of the head and neck at a frequency still similar to that in Steps 1 and 2. Step 4: The rhythmical movement increased in speed, the ears were continuously flattened backwards, the eyes closed and the vibrissae vibrated very rapidly. Step 5: The duration of the behavior in Step 4 increased, thus being the same behavior as Stage I. Step 6 is full seizure. In some cases, the animals showed behavior of both Step 3 and Step 4 and the results of these steps are combined. ( $\$$ ) female, $(\delta)$ male. 
The duration of the behavior at Step 4 increased that being the same behavior as Stage I. The animals which step 1 behavior had not detected showed the step 2 behavior as the first detectable behavior.

In contrast, the twenty-four sr animals which were subjected to the same treatment as that for the ss animals, including the stimulation by the S-method once a week, did not show the behaviors of any of these steps including the rhythmical movement of the pinnae. The R-method, which was applied to 2 sr animals during the period from 3 to 6 months of age, also failed to elicit these behaviors.

To ascertain the reason why the rhythmical movement of the pinnae was not induced in the sr animals, the facial nerve which induces the movement of the pinnae was studied macroscopically.

In both strains, facial nerve fibers in a bundle stained by the KB method were sparse in the brainstem, which made it easy to follow their pathway from the most dorsal area to their exit from the brainstem. The bundle consisted of several fascicles going ventrally and laterally (Fig. 2A, B). The pathway near the facial nuclei was difficult to detect by this method although the cells from which the fibers were originated were demonstrated in the tracing experiments with HRP to be the HRP-positive cells in the facial nuclei which were located as reported in [11]. When the fascicles exited the brainstem, they combined with a very thin fascicle which exited at a slightly more posterior location to form a thick nerve bundle. This nerve bundle ran laterally and a little anteriorly as described previously $[8,9]$ as far as the knee where it met the geniculate ganglion and changed its direction laterally and posteriorly running along the same pathway as the fibers originating from or passing through the geniculate ganglion (probably so-called intermediate nerve) thus forming a thick bundle (Fig. 2C, D). In the gerbil, the nerve fibers going in the geniculate ganglion tended to appear thinner, at least near the ganglion, than those of the so-called motor trunk and were discriminated from it. After separation of a branch going to the inner ear muscle, the facial nerve came up near the skull, where it changed its direction to become vertical. Along its course, it became incompletely encircled with bone. Then, the bundle of the vagus nerve, which probably originated mostly from the superior and inferior ganglions, ran within the posterior wall of the skull and passed through the facial nerve bundle horizontally contacting with it (Fig. 3B). The facial nerve went further vertically and after exiting from the skull, extended a branch which ran posteriorly and laterally toward the posterior
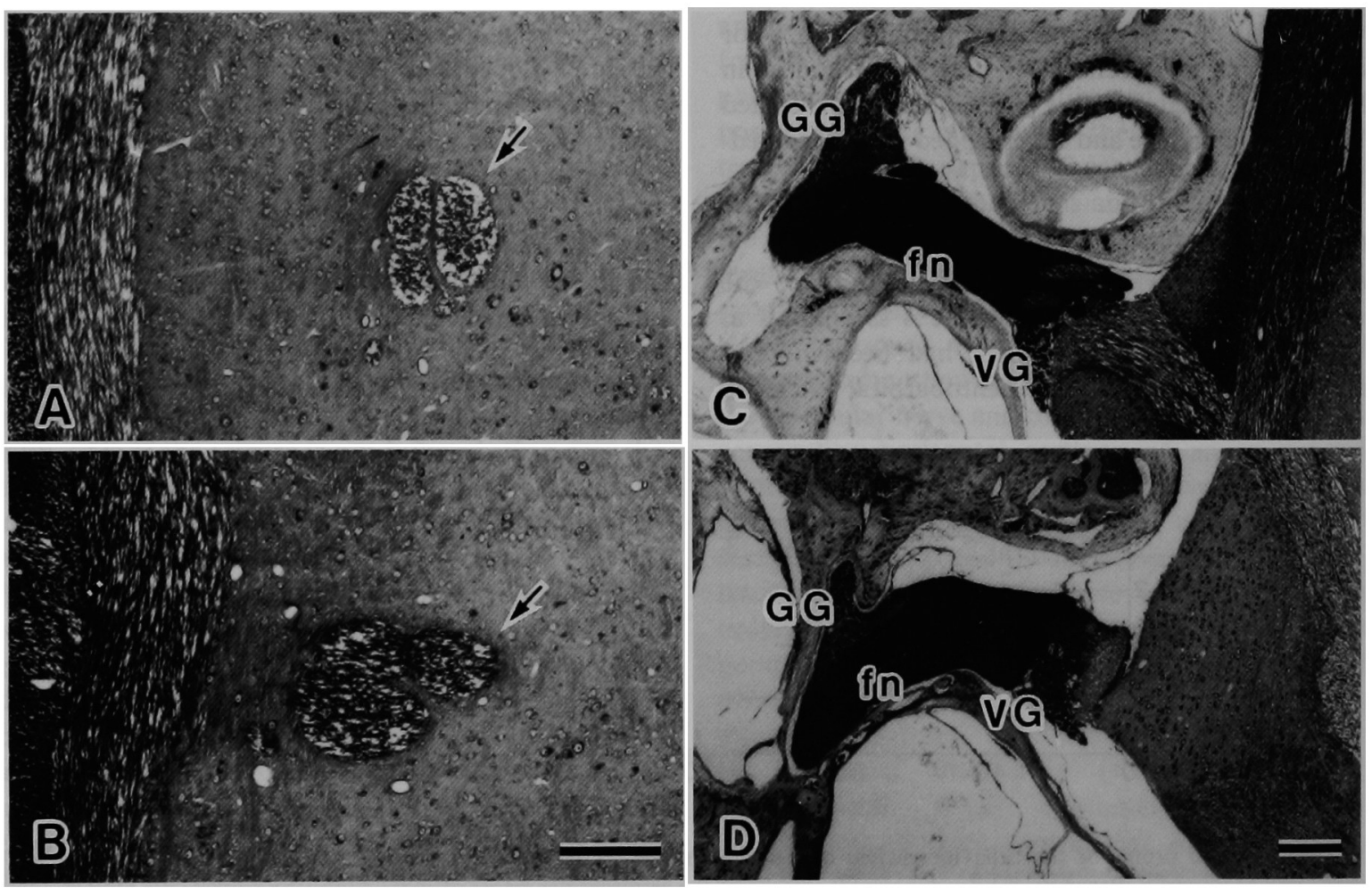

Fig. 2. Profiles of the facial nerve in the brainstem and those near the geniculate ganglion. The facial nerve bundle (arrow) was detectable in the brainstem because the nerve fibers were sparse in both ss (A) and sr (B) animals. In the horizontal sections stained by the KB method, the number of fascicles observed varied with the site but was mostly from 2 to 4 . top: anterior; left: lateral.

The facial nerve bundle which probably consisted mostly of the motor nerve exited from the brainstem, ran laterally and a little anteriorly with the intermediate nerve and turned its direction laterally and posteriorly at the geniculate ganglion in both ss (C) and sr (D) animals. The horizontal sections were stained by the KB method. top: anterior; left: lateral. fn, facial nerve (presumptive motor trunk); GG, geniculate ganglion; VG, vestibular ganglion. $\mathrm{Bar}=200 \mu \mathrm{m}$. 


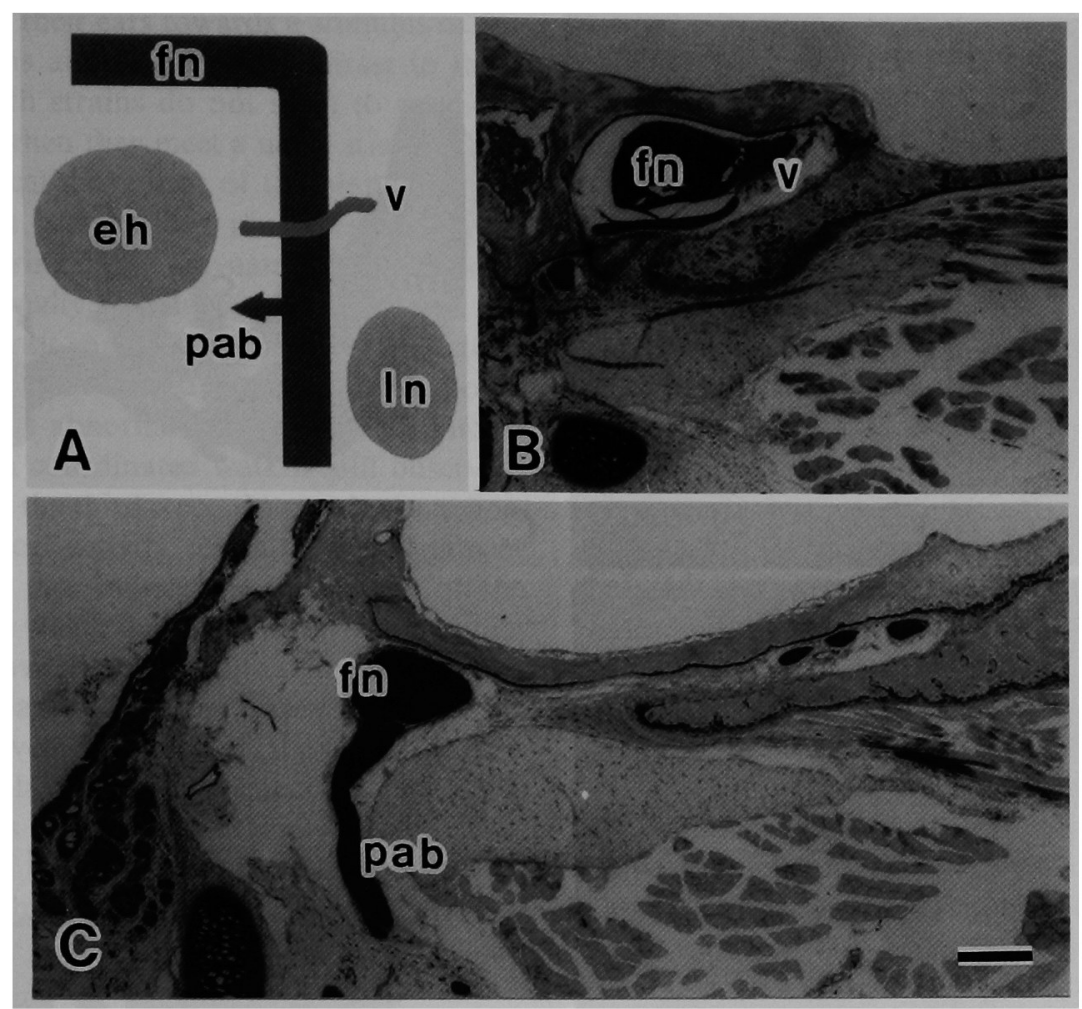

Fig. 3. Profiles of the facial nerve extending vertical along the outside of the skull. The diagram of the facial nerve bundle extending downwards is drawn schematically (A). The profile of the site where a vagus nerve bundle passes through or it may correspond to the site extending a communicating branch to the auricular branch of the vagus nerve $(\mathbf{B})$ and the site where a posterior auricle branch separates from the major bundle (C) are shown. (B) and (C) are the figures of the horizontal sections of an ss animal stained by the KB method but those of an sr animal are essentially the same. For (B) and (C), left: anterior, bottom: lateral. eh, ear hole; fn, facial nerve; ln, lymph node; pab, posterior auricular branch of the facial nerve; v, vagus nerve. Bar $=200 \mu \mathrm{m}$.

end of the ear hole (Fig. 3C). This posterior auricular branch went around the cartilage, and climbed on or into the posterior auricle muscle, splitting into several portions. These finally ended in the muscle (Fig. 4A, B).

Electrophysiological experiments showed that electrical stimulation of the area containing this posterior auricle muscle elicited backward movement of the pinnae, while stimulation of other areas around the ear hole caused the movement in different directions in young animals.

A secondary branch which had separated from the posterior auricle branch was found to go inside the cartilage and climb within it, splitting into several thin bundles. Some of these bundles appeared to end in the matrix.

All these features of the facial nerve were similarly observed in both ss and sr animals. The distances between the point where the nerve curved vertically, the site where it was crossed by the vagus nerve and the site extending the posterior auricle branch were also similar in both strains.

In our limited number of samples, however, the spacial relationship between the facial and vestibular nerves with the vestibular ganglion varied amongst the strains. In the gerbil, the nerve fibers originating from or passing through the geniculate ganglion tended to be thinner than those of the so-called motor trunk and were discriminated from the latter especially near the ganglion. In the ss animals, not a small portion of the upper part of the so-called motor trunk of the facial nerve bundle appeared to traverse the anterior part of the vestibular ganglion, where the nerve fibers extended into the ampullary crista of the lateral semicircular canal and the macula of utricle. On the other hand, in the sr animals, the ratio of the portion of the apparent motor trunk which traversed the vestibular ganglion was much less and it passed through the middle part of the vestibular ganglion which extended no nerve fibers to the vestibular end-organs. A three-dimensional structure in the ss animal was reconstructed and is shown in Fig. 5 .

\section{Discussion}

The results of this study show that a posture change induces the bilateral and synchronized rhythmical movement of the pinnae in the young ss animals. These movements seem to be limited at first to the pinnae although at this range of ages, the vibrissae move rapidly and almost continuously and the presence of some weak rhythmical movement with a rather low frequency as observed in the ear, even if occurring, may not be detected. There are many animal species including the cat, the rabbit and the dog 


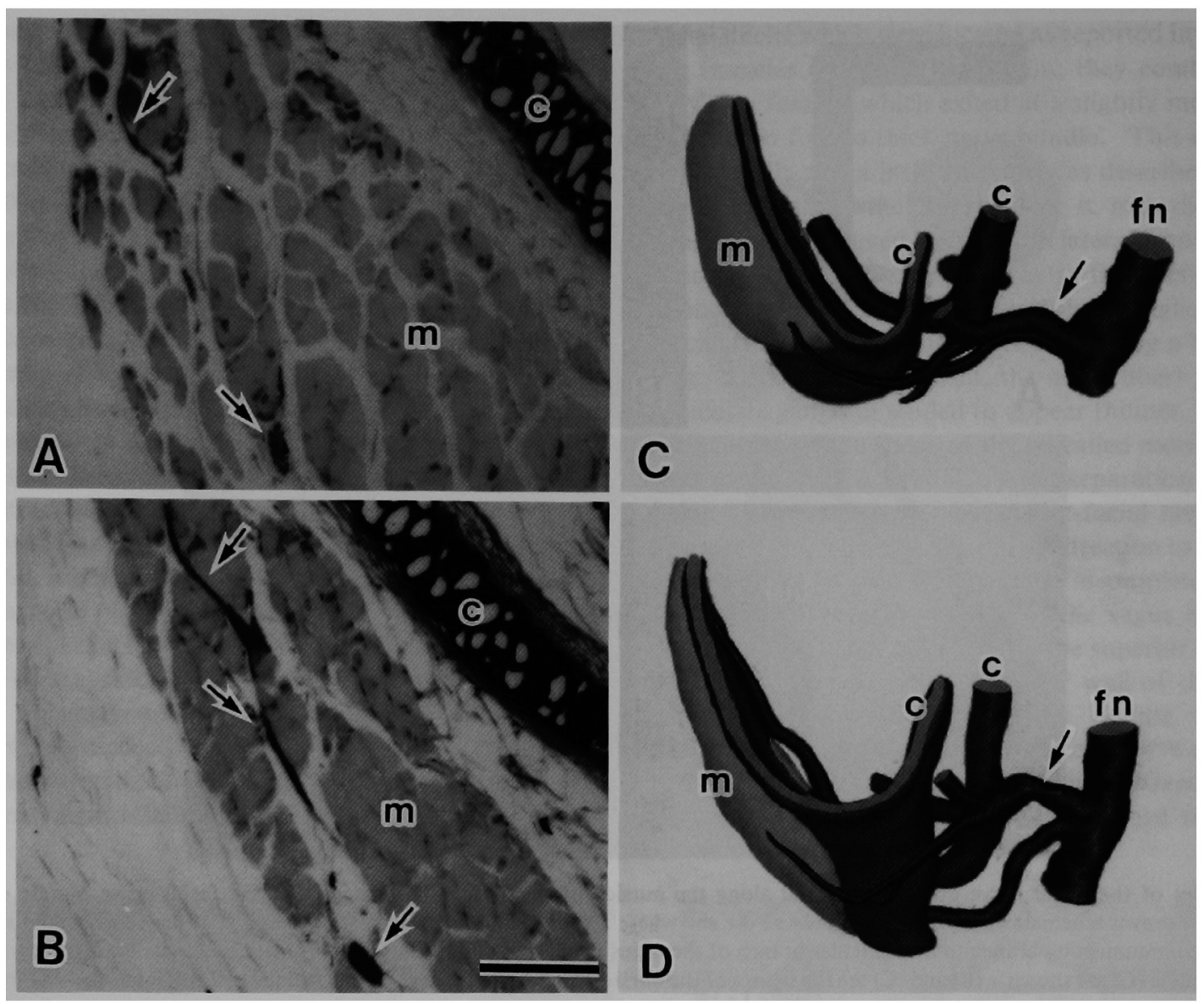

Fig. 4. The posterior auricle branch of the facial nerve bundle. The profile of the distribution of the posterior auricle branch of the facial nerve (arrows) in the muscle is shown in the horizontal sections stained by the KB method. (A): ss animal, (B): sr animal, top: anterior, left: lateral. Bar $=100 \mu \mathrm{m}$.

Three-dimensional images of the facial nerve made up as described in "Materials and Methods" are shown. (C): ss animal, (D): sr animal. The major bundle extends downwards and the posterior auricle branch (arrow) separates from it and climbs up the posterior auricle muscle. top: dorsal, left: lateral. c, cartilage; fn, facial nerve; m, posterior auricle muscle.

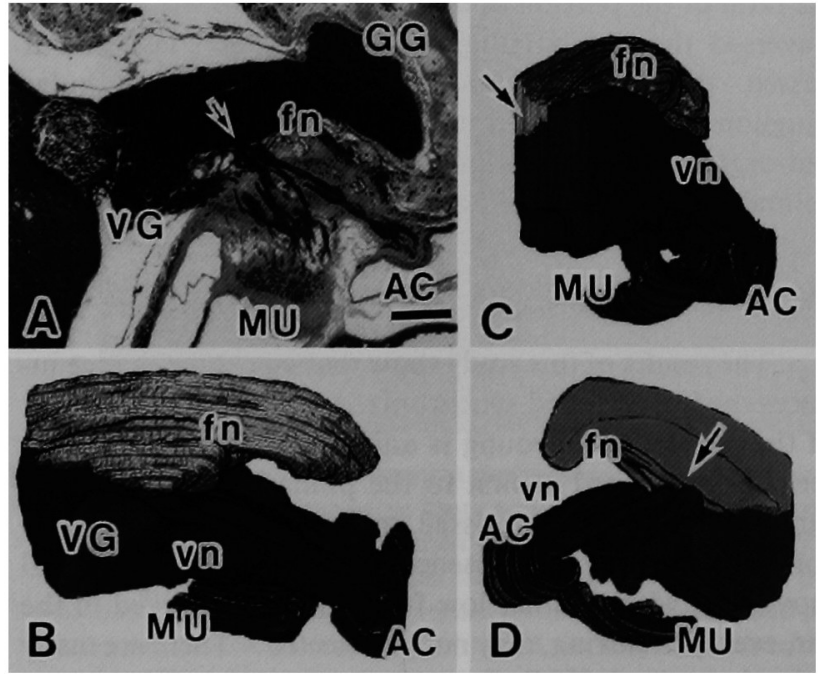

Fig. 5. Three-dimensional relationship between the facial nerve and the vestibular nerve. A three-dimensional structure showing the spacial relationship between the facial nerve and the vestibular nerve is reconstructed as described in "Materials and Methods". One of the original figures of the horizontal sections which is reversed in print to facilitate understanding is shown in (A). (B) shows the orientation similar to that in $(\mathbf{A})$ while $(\mathbf{C})$ is a figure rotated by approximately 90 degrees to show how the facial nerve appears to invade the vestibular bundle (arrow) and (D) is one rotated further to show that the fibers to the ampullary crista of the lateral semicircular canal and to the macula of utricle seem to come from the facial nerve bundle (arrow). AC, ampullary crista of the lateral semicircular canal; fn, facial nerve (so-called motor nerve trunk) with intermediate nerve; MU, macula of utricle; VG, vestibular ganglion; vn, vestibular nerve. Vestibular ganglion is included in the area of "vn" in (C) and (D). In (A) and (B), top: anterior, right: lateral. In (B), the top of the piled structure corresponds the most dorsal section. Bar in (A) $=200 \mu \mathrm{m}$. 
which normally orient their ears towards a stimulus or for expression of emotions and so on. In contrast to these species, gerbils of both strains do not seem to normally move their ears even when they meet a novel stimulus and therefore the rhythmical movement of the pinnae elicited in the sensitive strain is a curious phenomenon in this animal species. During this movement, the animals appeared to walk normally, which seems to show that the abnormal event does not spread into the nervous systems which regulate the movement of other parts of the body. At the second step, the abnormal rhythm spreads into at least the neck while a coordinated walk is still observed, although this is lost at the third step. In step 2, the abnormal electrophysiological activity seems to spread into at least the area which can induce the movement of the neck. The animals in which the step 2 behavior is the first detectable one may have the kind of brain in which abnormal activity spreads easily into these areas, not localizing at the area for ear movement, however, the possibility that some weak and short-lasting ear movement escaped observation in the previous test remains.

The frequency of the movement increases and finally, the ears remain lying backwards, the eyes remain shut and the vibrissae tremble vigorously. These sequential events seem to suggest that the rhythmical backwards movement of the pinnae is an early, if not the first, event in the establishment of epileptogenesis. It may be an important step for later development of epileptic behaviors.

In the "kindling model", a well-known experimental model of epilepsy, a weak stimulus given repetitively makes the brain epileptic and abnormal behavior develops step by step increasing in severity [4] much like the behaviors observed in the gerbil. For the development of epileptic behavior in this model, afterdischarge that is often composed of rhythmical waves and continues after the cessation of the stimuli for several to several tens of seconds, is required. Considering this model, there is a possibility that the rhythmical movement of the pinnae of the gerbil is elicited by short-lasting rhythmical afterdischarge which occurs in the cerebral cortex after the posture change. To evaluate this, an electrophysiological study combined with an anatomical one is currently being performed in our laboratory.

In contrast, the sr animals do not show any of the epileptic behaviors observed in the ss animals even though they were treated in exactly the same way, including stimulation by the S-method, as the ss animals and were also subjected to the R-method, a more complex method. There may be some difference in the component(s) in the neuronal pathway between the site receiving the stimulation and the site responsible for resultant behavior, in this case, the rhythmical movement of the pinnae. The most simple idea is that the pinnae cannot be moved because of an abnormality of the facial nerve innervating the ear muscle, such as its absence and this abnormality, in turn, causes the abortion of the development to later behaviors. Thus, as a first step in clarifying the reason behind the difference, the facial nerve was studied macroscopically.

The facial nerve bundle left the brainstem, ran laterally and slightly anteriorly, and changed its direction at the geniculate ganglion posteriorly and laterally. After it reached near the skull, it changed its direction to become vertical and extended a branch to the posterior auricle muscle. This fundamental profile is similar to that of other mammalians including the dog and man $[5,10]$ although details such as the type of nerves in the intermediate nerve bundle or the presence of the facial motor nerve passing through the geniculate ganglion $[2,14]$ as reported for other species were not examined in this study. In man, the facial nerve extends a branch called "a communicating branch" to the auricular branch of the vagus nerve [14]. In the gerbil, a part of the facial nerve seemed to join with what appeared to be the branch of the vagus nerve crossing the major part of the facial nerve bundle but tracing experiments were required to ascertain this point. The profile of the nerve fibers in the muscle is also similar in both gerbil strains. Therefore, it is unlikely that the different behavioral responses of the resistant animals is due to a macroscopical abnormality such as an absence of the facial nerve which innervates the ear muscle. These two strains probably have difference(s) in components other than the facial nerve although the possibility of a functional difference in the facial nerve remains.

Within the limits of our small number of samples, a closer spacial relationship between the so-called motor fiber bundle of the facial nerve and the vestibular one was observed in the ss animals. To evaluate this, tracing experiments combined with an electrophysiological study with more animals are required, which is difficult at present because of the small colony of the resistant strain. It may be only individual differences but if this finding reflects the true difference between the strains, then it may be interesting since the major stimulus during the posture change seems to be a vestibular one. In the rabbit, posture change induced not only an eye but also an ear reaction [13] and in the cat, the direct projection from vestibular nuclei to facial nucleus was reported as a system for the coordination of movement to orient attention toward a stimulus [16]. The ss animals do not use their ears for attention, but there may be some close relationship between the vestibular system and facial one which is activated together with the cerebral cortex, such as the vestibular area [6], under some conditions.

Further experiments including an electrophysiological study, an ultrastructural analysis and some biochemical study such as the analysis of the components in the neurotransmission are required to clarify the differences between these two strains which in turn may contribute to an understanding of epileptogenesis. 


\section{Acknowledgments}

We are grateful to Image and Measurement, Inc (Tokyo, Japan) for help with the three-dimensional reconstruction with the program "VoxBlast" and acknowledge the fruitful discussions from Drs. $\mathrm{N}$. Takeda, S. Horii (Osaka University), S. Saga (Aichi Medical University), and A. Mizutani. M. Oshima helped hand-made reconstruction. This study was supported in part by a Grant-in-Aid for Scientific Research from the Ministry of Education, Science and Culture of Japan (No. 07640917).

\section{References}

1. Adams, J. C.: Heavy metal intensification of DAB-based HRP reaction product. J. Histochem. Cytochem. 29; 776, 1981.

2. Anson, B. J.: Morrs' Human Anatomy, McGraw-Hill Book Company, N.Y., 1966.

3. Buckmaster, P.S., Tam, E. and Schwartzkroin, P. A.: Electrophysiological correlates of seizure sensitivity in the dentate gyrus of epileptic juvenile and adult gerbils. J. Neurophysiol. 76; 2169-2180, 1996.

4. Goddard, G. V., McIntyre, D. and Leech, C.: A permanent change in brain function resulting from daily electrical stimulation. Exp. Neurol. 15; 295-330, 1979.

5. Guerrier, Y.: Surgical anatomy, particularly vascular supply of the facial nerve. In "Facial Nerve Surgery", ed. by U. Fisch, Kugler Medical Publications B.V., Amsterveen, 1976, pp. 1323.

6. Ito, M. and Seto-Ohshima, A.: The site of cortical utricular representation with special reference to the somatosensory barrelfield in the gerbil. Ann. Otol. Rhinol. Laryngol., 1997. (in press)

7. Jobe, P. C., Mishra, P. K., Ludvig, N. and Dailey, J. W.: Scope and contribution of genetic models to an understanding of the epilepsies. Critical Rev. Neurobiol. 6; 183-219, 1991.

8. Kawamura, N., Seto-Ohshima, A. and Ito, M.: Computerassisted three-dimensional reconstruction of the vestibular end-organs of the gerbil using the serial paraffin sections. Acta Histochem. Cytochem. 30; 117-120, 1997.

9. Kawamura, N., Ito, M. and Seto-Ohshima, A.: Postnatal development of the structure of the peripheral vestibular system of the gerbil. Acta Histochem. Cytochem. 30; 1997. (in press)

10. Miler, M. E.: Anatomy of the Dog, W. B. Saunders Company, N.Y., 1964.

11. Park, H. J., Kim, H. N. and Kim, K. M.: Redistribution of facial nerve motor neurons after recovery from nerve crushing injury in the gerbil. Acta Otolaryngol. 115; 273-275, 1995.

12. Ralis, H. M., Beesley, R. A. and Ralis, Z. A.: Techniques in Neurohistology, Butterworths \& Co. (Publishers) Ltd., London, 1973.

13. Schaefer, K.-P., Meyer, D. L. and Schott, D.: Optic and vestibular influences on ear movements. Brain Behav. Evol. 4; 323-333, 1971.

14. Semba, K. and Egger, M. D.: The facial "motor" nerve of the rat: control of vibrissal movement and examination of motor and sensory components. J. Comp. Neurol. 247; 144-158, 1986.

15. Seto-Ohshima, A., Ito, M., Kudo, T. and Mizutani, A.: Intrinsic and drug-induced seizures of adult and developing gerbils. Acta Neurol. Scand. 85; 311-317, 1992.

16. Shaw, M. and Baker, R.: Direct projections from vestibular nuclei to facial nucleus in cats. J. Neurophysiol. 50; 1265$1280,1983$.

17. Takeuchi, Y. K., Takeuchi, I. K., Murashima, Y. and SetoOhshima, A.: Age-related appearance of dystrophic axon terminals in cerebellar and vestibular nuclei of Mongolian gerbils. Exp. Anim. 46; 59-65, 1997. 